\title{
Un Proceso de Modelización de la Membrana Celular en la Formación del Profesorado en Biología en la Universidad
}

\section{A Process of Modeling the Cell Membrane in Biology Teacher Training at University}

\author{
iD Eduardo Enrique Lozano' \\ Agustín Adúriz-Bravo² \\ iD Nora Bahamonde ${ }^{1}$
}

'Universidad Nacional de Río Negro, Centro de Estudios e Investigación en Educación (CEIE), Viedma, Río Negro, Argentina. 2Universidad de Buenos Aires, Facultad de Ciencias Exactas y Naturales, Instituto de Investigaciones en Didáctica de las Ciencias Naturales y la Matemática (CeFIEC), Buenos Aires, Argentina.

Autor correspondiente: elozano@unrn.edu.ar

Resumen: El estudio se contextualiza en la formación inicial del profesorado en Biología en el ámbito de la Universidad, en una línea de trabajo que propone que los espacios de formación disciplinar en biología que cursan los estudiantes deben estar informados e intervenidos por modelos y estrategias de enseñanza provenientes de los espacios de formación en didáctica de las ciencias naturales. Se presentan resultados de un proceso de modelización de la membrana celular a partir del análisis de un hecho científico. Se caracterizan los modelos de partida de los estudiantes, el proceso de modelización intermedia y los modelos de arribo, orientados a los aspectos de fluidez y autoensamblaje de las moléculas que componen la membrana. El análisis de las producciones nos permite inferir que los modelos iniciales que construyeron para la situación propuesta se fueron reelaborando y complejizando, y dan cuenta de una reconstrucción teórica del fenómeno, anclándolo en el modelo científico de referencia.

Palabras clave: Enseñanza de biología; Membrana celular; Formación del profesorado; Enseñanza superior.

Abstract: The study is contextualized in a University teacher training program of Biology, in a perspective that proposes that disciplinary training spaces in biology developed by the students should be informed and intervened by models and teaching strategies coming from training spaces in the didactics of natural sciences. Results of a cell membrane modeling process are presented based on the analysis of a scientific fact. Initial models by the students are characterized, as well as the process of intermediate modeling and the structuring of the membrane model, in particular the aspects of fluidity and self-assembly of the molecules that compose it. The analysis of the productions allows us to infer that the initial models made for the proposed situation were becoming more complex and allowed for the theoretical reconstruction of the phenomenon, anchored in the scientific reference model.

Keywords: Biology teaching; Cell membrane; Teacher training; Higher education.

Recibido en: 27/03/2019

Aprobado en: 30/04/2020 


\section{Introducción}

La modelización de fenómenos (GILBERT; JUSTI, 2016) y la construcción de ideas metacientíficas (LEDERMAN, 2006) son directrices teóricas actuales del campo de la didáctica de las ciencias naturales, y se complementan con la perspectiva de abordaje de asuntos sociocientíficos (ZEIDLER et al., 2005), que permiten contextualizar y dar sentido y relevancia a las producciones de la ciencia escolar que llevan a cabo los estudiantes (IZQUIERDO et al., 1999). La investigación que desarrollamos, inscripta en la línea: modelos y modelización (GILBERT; OSBORNE, 1980), puso en valor ciertos aspectos de esas directrices al interior de un espacio de formación disciplinar del profesorado, la asignatura Biología Celular, al implementar una Unidad Didáctica (UD) orientada a la modelización de los aspectos de fluidez y autoensamblaje de la membrana celular a partir de la problematización sobre un hecho científico vinculado con la manipulación de células. Los objetivos fueron: describir los modelos de partida, intermedios y de arribo de los estudiantes e interpretar los procesos de modelización. Una nota distintiva de este estudio es que, en el diseño de la unidad didáctica, se integraron actividades para favorecer y potenciar, en paralelo, la modelización disciplinar y la construcción de una idea clave metacientífica sobre el carácter analógico de los modelos, con resultados que no se incluyen en este artículo (LOZANO; BAHAMONDE; ADÚRIZ-BRAVO 2016).

La elección del modelo de membrana celular, en particular de las propiedades de fluidez y autoensamblaje que le dan las moléculas que la estructuran, se justifica en virtud de que esos aspectos del modelo están en la base de la comprensión de núcleos de interés en biología, como los nuevos modelos teóricos sobre el origen de las primeras células y la evolución de los sistemas de endomembranas (RAUCHFUSS, 2008) y también en el desarrollo actual de tecnologías, como líneas de productos farmacológicos que utilizan liposomas artificiales para transportar sus principios activos, en la manipulación de células con diferentes objetivos, entre otros temas. Sin embargo, se advierte la escasa significatividad que suelen tener para los estudiantes esos aspectos del modelo a la hora de explicar fenómenos biológicos en los que podrían implicarse; por ejemplo, pueden definir la membrana como un 'mosaico fluido', pero no pueden justificar con ese modelo, que implica la propiedad de autoensamblaje de los fosfolípidos, el transporte de una proteína de exportación por diferentes y sucesivas vesículas hacia la membrana celular, o explicar cuestiones más sencillas: el fenómeno que le permite a una ameba unir sus pseudópodos para atrapar una presa.

\section{Modelos y Modelización en la Enseñanza de la Membrana Celular}

La naturaleza de los modelos ha sido abordada tanto en el campo de la psicología cognitiva como en el de la filosofía de la ciencia (GILBERT; JUSTI, 2016); el concepto de representación (NERSESSIAN, 2002) es el que articula las miradas de estas dos disciplinas y está en la base de las implicancias que tiene la utilización de modelos en la enseñanza de las ciencias. Para nuestro trabajo, y desde una visión semántica de las teorías científicas (ADÚRIZ-BRAVO, 2012), adoptamos la caracterización que hace Giere (1988) sobre los 
modelos, al considerarlos entidades abstractas, representaciones que subrogan aspectos del mundo bajo estudio y que actúan como mapas para facilitar su comprensión. En el esquema de Giere (1988) de modelo teórico, la relación entre los modelos y la realidad se establece en términos de "similaridad", y esto implica que representación y realidad se vinculan analógicamente en forma más o menos estrecha. Respecto de las posibilidades de definir los modelos teóricos, el autor sostiene que esto puede hacerse mediante diferentes formas de lenguaje -enunciados, gráficos, analogías, etc., y no solamente con sistemas axiomáticos.

Figura 1 - Concepción de modelo teórico para el desarrollo de la Unidad Didáctica

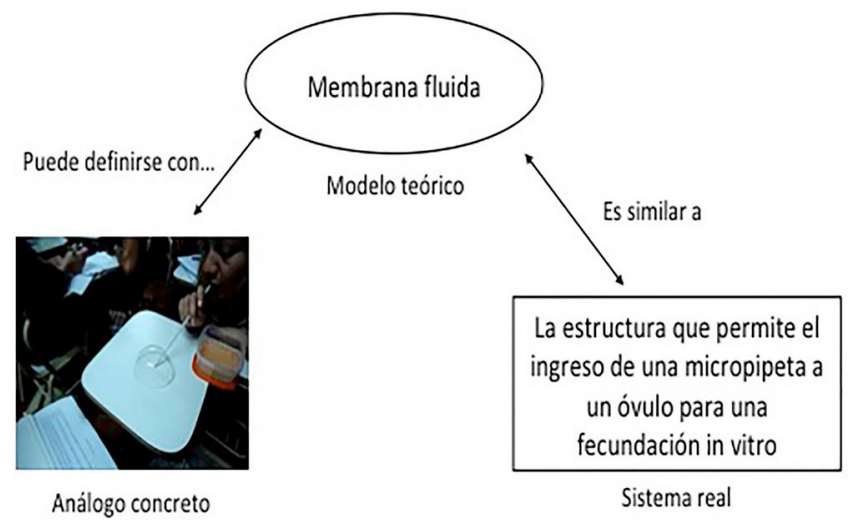

Fuente: Adaptado de Giere (1988).

En cuanto a la modelización en la enseñanza de las ciencias (CLEMENT, 2000; GILBERT; OSBORNE, 1980; GUTIÉRREZ, 2014; JUSTI, 2006; KHAN, 2011; MARBA, 2014), la concebimos como un proceso que implica inicialmente la elicitación de los modelos mentales de los estudiantes, expresados mediante diferentes representaciones, y que luego transcurre por medio de avances y recursividades para poner a prueba esos modelos, evaluarlos, criticarlos y modificarlos al aplicarlos a la explicación y comprensión de situaciones específicas (GILBERT; JUSTI, 2016; GÓMEZ, 2005). Un objetivo central de la modelización es que los hechos del mundo abordados sean reconstruidos teóricamente al interior de la clase y alcancen un elevado nivel de significatividad (IZQUIERDO et al., 1999).

Desde esta perspectiva, y en particular para la enseñanza de la biología orientada al estudio de los organismos, se han desarrollado propuestas de modelización a nivel de organización de sistemas (BUCKLEY, 2000; BAHAMONDE; GÓMEZ GALINDO, 2016) y también a nivel celular (CLÉMENT, 2007; DÍAZ DE BUSTAMANTE; JIMÉNEZ ALEIXANDRE, 2008; MENGASCINI, 2006; RODRÍGUEZ PALMERO; MOREIRA, 1999; VERHOEFF, 2003). En el contexto de diferentes investigaciones y experiencias de enseñanza para favorecer procesos de modelización de la membrana celular en las clases de ciencias (IDÁRRAGA, 2015; LAYING THE FOUNDATION, 2004; NUFFIELD FOUNDATION, 2001; UNIVERSITY OF UTAH, 2008), la unidad didáctica (UD) que implementamos aborda la estructura fluida y autoensamblante de la membrana celular, ya que es el aspecto que permite dar sentido al hecho científico abordado: la fecundación in vitro, y se investiga el ciclo de modelización protagonizado por los estudiantes. 
Ese ciclo incorpora la utilización de mediadores simbólicos y análogos concretos, uno de ellos referenciado en un episodio de la historia de la construcción del modelo de membrana. El énfasis puesto en el desarrollo de la modelización intermedia y en la elaboración de modelos de arribo, y el tiempo destinado al desarrollo de la UD para que pudiera implementarse de manera significativa, creemos que constituyen un avance en algunos de los problemas que han sido referenciados en el campo de la investigación y de la enseñanza de las ciencias basada en modelos (KHAN, 2011) y en particular en la modelización de la membrana celular constatada en los antecedentes. Luego, en la continuidad de la formación que reciben los estudiantes, y para dar sentido a otros fenómenos que lo requieren, las modelizaciones incorporan a las proteínas de membrana.

\section{Metodología}

La investigación que llevamos a cabo sobre la modelización adoptó un enfoque metodológico de tipo cualitativo (TAYLOR, 2014). Los datos surgieron por un tipo especial de intervención del docente-investigador, mediante el diseño y la implementación de una Unidad Didáctica (UD) en el contexto natural en el cual desarrolla sus clases de Biología celular en la formación del Profesorado. Afín a las perspectivas del diseño de la Actividad Científica Escolar (ACE) (SANMARTÍ, 2002) y los Estudios de Diseño (DBR) (REIGELUTH; FRICK, 1999), se propuso un trabajo de elaboración, aplicación y evaluación de una secuencia de enseñanza en un ciclo iterativo, alimentado a partir de los propios datos de la investigación (PSILLOS; KARIOTOGLOU, 2016). La primera etapa, Fase preliminar, implicó el diseño de la UD, su aplicación en el aula y el análisis y evaluación de su implementación. La segunda etapa, Fase definitiva, implicó el ajuste y rediseño de la UD a partir de los datos obtenidos, su nueva aplicación en el aula con otro grupo de estudiantes al año siguiente, y el análisis final de los datos. Los resultados que se exponen en este artículo se elaboraron a partir de los datos obtenidos en la Fase definitiva, sobre las producciones de 32 estudiantes distribuidos en siete grupos de trabajo.

Como unidades de análisis, las que constituyeron el corpus documental de la investigación, se consideraron: producciones de gráficos y textos escritos (individuales en la actividad de modelización inicial y luego grupales en el resto de la modelización), episodios del trabajo grupal, interacciones con materiales, intercambios y discusiones, a partir de grabaciones/videos o tomando nota en el cuaderno de registros. La modelización inicial individual permitió que todos los estudiantes representaran el fenómeno analizado y dio lugar a la caracterización de los modelos de partida que se describen en los Resultados. Luego, la continuidad de la modelización en el ámbito grupal permitió la comunicación de los modelos iniciales y diferentes procesos de negociación y regulación en el cual algunas ideas, conceptos o aspectos de los modelos, fueron descartados y otros seleccionados (IZQUIERDO et al., 1999). En el análisis de la modelización se establecieron diferentes categorías que reunían un grupo de elementos bajo un título genérico y que otorgaban un sentido generado desde la intervención teórica sobre los datos (BARDIN, 1986). La tarea de definición de las categorías se efectuó en reuniones entre el docente investigador y el equipo de dirección del proyecto. Las categorías construidas se presentan en la sección resultados. 


\section{El Diseño de la Unidad Didáctica}

El diseño de la unidad didáctica se llevó a cabo según las orientaciones generales de la Actividad Científica Escolar (SANMARTí, 2002), e implicó la elaboración de actividades para el desarrollo de las diferentes etapas de la modelización. Estas actividades se fueron desarrollando en seis sesiones de clases. En este artículo se desarrollan sólo los resultados de las sesiones 1, 2 y 3, referidas a la modelización inicial, intermedia y de arribo. Con el objeto de mostrar la naturaleza integradora de la unidad didáctica se incorporó al cuadro 1 las consignas de la sesión 4, vinculadas al eje metacientífico, si bien no se desarrollan resultados (LOZANO; BAHAMONDE; ADÚRIZ-BRAVO, 2016). Las sesiones 5 y 6 , de formalización del modelo y aplicación, no fueron incluidas en el cuadro 1.

Cuadro 1 - Unidad didáctica rediseñada para la fase definitiva

Unidad Didáctica sobre membrana celular y modelo analógico: fase definitiva

Sesión 1: Formulación del problema y modelización inicial individual

En un caso de fertilización in vitro, ¿por qué una micropipeta puede entrar y salir del óvulo sin producirle daños en su estructura, ya que luego deberá ser implantado en el útero de una mujer? Piense y explique mediante gráficos y textos explicativos y con todo el detalle que pueda.

Toma de datos: recolección de dibujos y textos individuales

Sesión 2: Discusión de los modelos iniciales al interior de cada grupo: introducción de mediadores analógicos

a. Organizados en grupo compartan las diferentes explicaciones que dieron al inicio. Observen el siguiente video que muestra imágenes de una fecundación in vitro de un óvulo a microscopio óptico. ¿Les aporta nuevos elementos a las discusiones?

Toma de datos: recolección de discusiones grupales

b. Con qué materiales concretos ustedes podrían ejemplificar ahora el caso del ingreso de la micropipeta al óvulo? ¿Por qué? (Se les ofrecerá a los alumnos una mesa con diferentes materiales: gelatina preparada, agua, aceite, jabón, globos, coladores, recipientes de plástico, sorbetes, pipetas de vidrio, pelotitas de telgopor)

Toma de datos: fotografías de los modelos

Sesión 3: Introducción de un mediador analógico ejemplar desde la historia de la ciencia y de un mediador simbólico (molécula de lípido anfipático): inicio de la representación gráfica del fenómeno a nivel molecular

a. Lean en grupos el siguiente texto (ver Anexo A)

b. Simulen el fenómeno del ingreso de la micropipeta al óvulo con una pompa y un sorbete. ¿Qué ocurre? Armen varias pompas contiguas, en contacto. ¿Qué ocurre entre ellas? Coloquen pequeñas gotitas de aceite separadas entre sí en un recipiente con agua y generen corrientes suaves que las pongan en contacto ¿Qué observan cuándo están próximas?

c. Ahora piensen esos fenómenos a nivel de las moléculas de lípidos anfipáticos que se encuentran en esos sistemas. Construyan gráficos a ese nivel y textos explicativos, que permitan explicar lo que han observado en cada caso.

Toma de datos: recolección de gráficos y textos complementaros. Grabación de discusiones al interior de los grupos.

Sesión 4: Reflexión sobre la utilización de los modelos

a. Durante el trabajo realizado hasta aquí fue frecuente la utilización de la palabra "modelo" para referirnos a las diferentes producciones que se fueron llevando a cabo. ¿Podrían tratar de definir a qué hemos llamado modelo en nuestras clases y por qué lo denominamos así?

b. Leer el material que se adjunta (texto extraído de Adúriz-Bravo, 2005) sobre analogías. ¿Qué lugar ocupa el modelo entre una hipótesis y la realidad? Vuelvan al texto de Quincke (KEPNER, 1979 apud HIDALGO; DEVÉS; LAGOS, 1996, p. 6). ¿Qué estrategia utilizó para explicar el límite de la célula?

Toma de datos: recolección de textos producidos por los estudiantes. Grabación de discusiones al interior de los grupos.

Fuente: Elaborado por los autores.

\section{Resultados}

\section{Modelos de partida}

En la Sesión 1, luego de presentada la situación problemática y del total de 32 alumnos, sólo en cuatro casos no fue posible identificar claramente una hipótesis en las respuestas. 
Entre los 28 considerados aptos, algunos alumnos ofrecieron más de una explicación, en algunos casos hasta cuatro, y todas las ideas han sido tenidas en cuenta en la sistematización de los datos. Al analizar las explicaciones respecto de por qué no se rompe el óvulo luego de introducir y sacar una micropipeta de él, se consideró apropiado organizarlas en tres categorías: 'sustancias', 'estructura porosa'y 'propiedades de la micropipeta'. Para la asignación de las dos primeras categorías a las explicaciones de los estudiantes se consideró adecuado tener en cuenta diferentes visiones de las relaciones estructura/función que sobre el límite de la célula se discutieron y confrontaron en el largo proceso de construcción del modelo de membrana celular, que ocupó una buena parte del siglo XIX y que fueron identificadas en el estudio histórico epistemológico llevado a cabo (LOZANO; BAHAMONDE; ADÚRIZ-BRAVO, 2016). Una de ellas aglutina la mayoría de las ideas, las cuales consideran que las razones que permiten comprender el fenómeno están en relación con la presencia de un tipo de sustancia presente en el límite de la célula y que cumpliría con el requisito de sellarse luego del procedimiento. En este grupo, y en el contexto de la situación problemática propuesta, sólo tres estudiantes activaron un modelo de membrana lipídica y sólo uno de ellos lo aplicó con funciones de fluidez. Otra categoría incluye ideas que no referencian sustancias sino un tipo de estructura con poros por los cuales podría ingresar la micropipeta, sin producir daños a la célula. La tercera categoría incluye ideas que no hacen referencia al límite de la célula sino a propiedades de la micropipeta como el tamaño o sustancias que puede tener sobre su superficie (cuadro 2).

Cuadro 2 - Modelos iniciales para explicar la situación problemática

\begin{tabular}{|c|c|}
\hline Categorías & Ejemplos de modelos individuales \\
\hline $\begin{array}{l}\text { Sustancias: } 20 \text { menciones, de las } \\
\text { cuales } 12 \text { hacen referencia a la gela- } \\
\text { tina, } 4 \text { al agua, } 1 \text { al aceite y } 3 \text { a lípidos } \\
\text { de membrana }\end{array}$ & 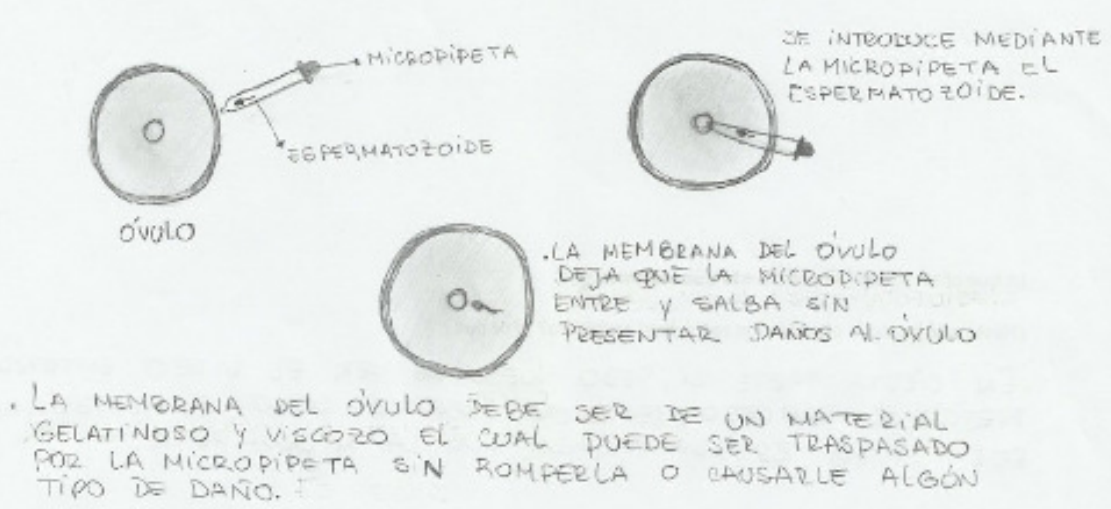 \\
\hline Estructura porosa: 10 menciones & 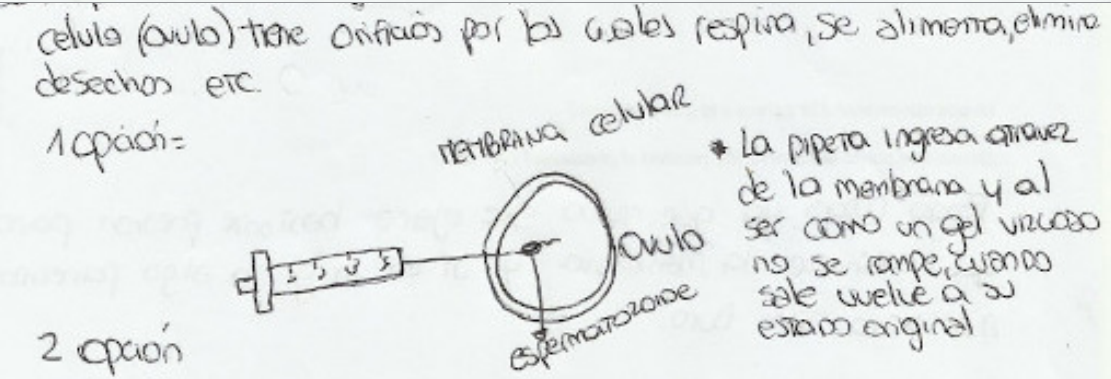 \\
\hline
\end{tabular}




\begin{tabular}{|c|c|}
\hline Categorías & Ejemplos de modelos individuales \\
\hline $\begin{array}{l}\text { Propiedades de la micropipeta: } 9 \\
\text { menciones }\end{array}$ & 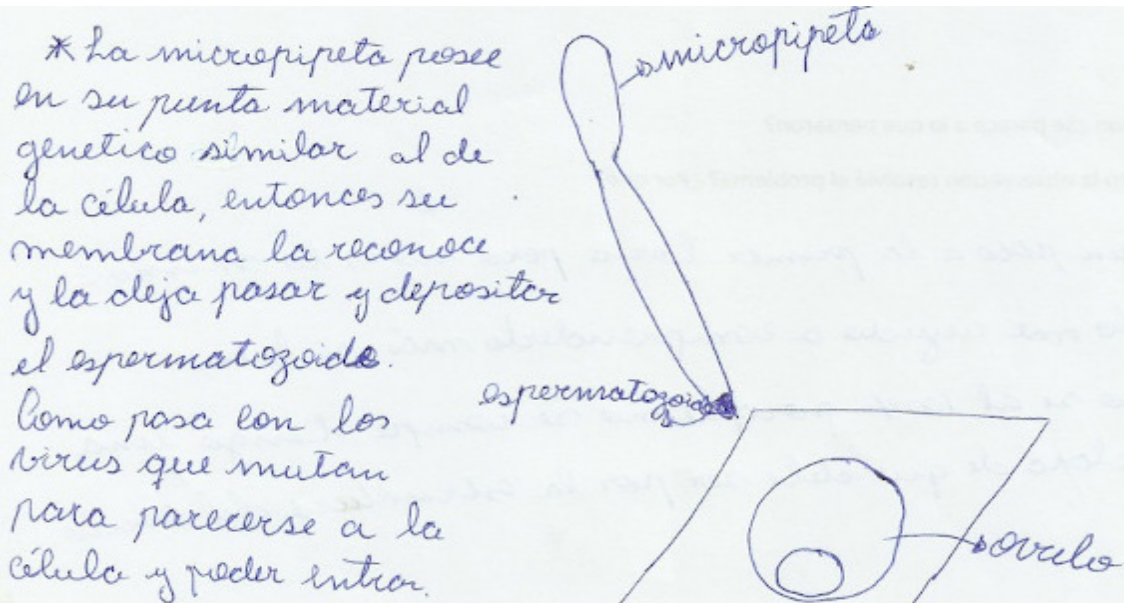 \\
\hline
\end{tabular}

Fuente: Elaborada por los autores a partir de la codificación de las producciones de los estudiantes.

\section{Modelización intermedia}

\section{a. Discusión de los modelos individuales al interior de cada grupo y construcción de un primer análogo material}

En el inicio de la Sesión 2, los integrantes de cada uno de los grupos compartieron sus gráficos y sus ideas iniciales respecto del fenómeno analizado y se proyectó un video breve que, a nivel de microscopio óptico, daba cuenta del ingreso y salida de una micropipeta a un óvulo dejando en su interior un espermatozoide. Esta actividad generó discusiones al interior de cada grupo y básicamente puso en cuestión el modelo de 'estructura porosa', ya que los alumnos reconocían que en el procedimiento no se observaba la búsqueda de un sitio especial para el ingreso de la micropipeta. Por otra parte, la observación de la manipulación técnica no daba cuenta de la presencia de sustancias sobre la micropipeta y la relación de tamaño entre óvulo y micropipeta y la presión ejercida sobre el límite de la célula para que ingresara, permitió cuestionar que el tamaño de la micropipeta fuese un factor significativo. (Por cuestiones de espacio en este artículo no se exponen producciones de los estudiantes sobre esta actividad de la Sesión 2).

La continuación del trabajo se orientó hacia la discusión sobre las hipótesis referidas a sustancias que habían aparecido en la mayoría de los modelos iniciales de los estudiantes.

Comenzaron a hacer pruebas con los diferentes elementos y sustancias presentes en la mesa de trabajo (gelatina preparada, agua, aceite, jabón, globos, coladores, recipientes de plástico, sorbetes, pipetas de vidrio, pelotitas de telgopor). Luego de la confrontación de algunas ideas iniciales con los materiales se fueron descartando hipótesis, como por ejemplo la analogía con la gelatina, ya que al ser intervenida con un sorbete claramente no producía un autosellado como el que esperaban. Tras un intenso debate al interior de cada grupo y en función de la consigna b de la Sesión 2 presentaron, en una puesta en común en clase, un análogo material que cumplía con las condiciones del fenómeno de ingreso y salida de la micropipeta al óvulo. 
Cuadro 3 - Modelos iniciales y modelos consensuados a posteriori en los grupos, que cumplen con las condiciones del fenómeno

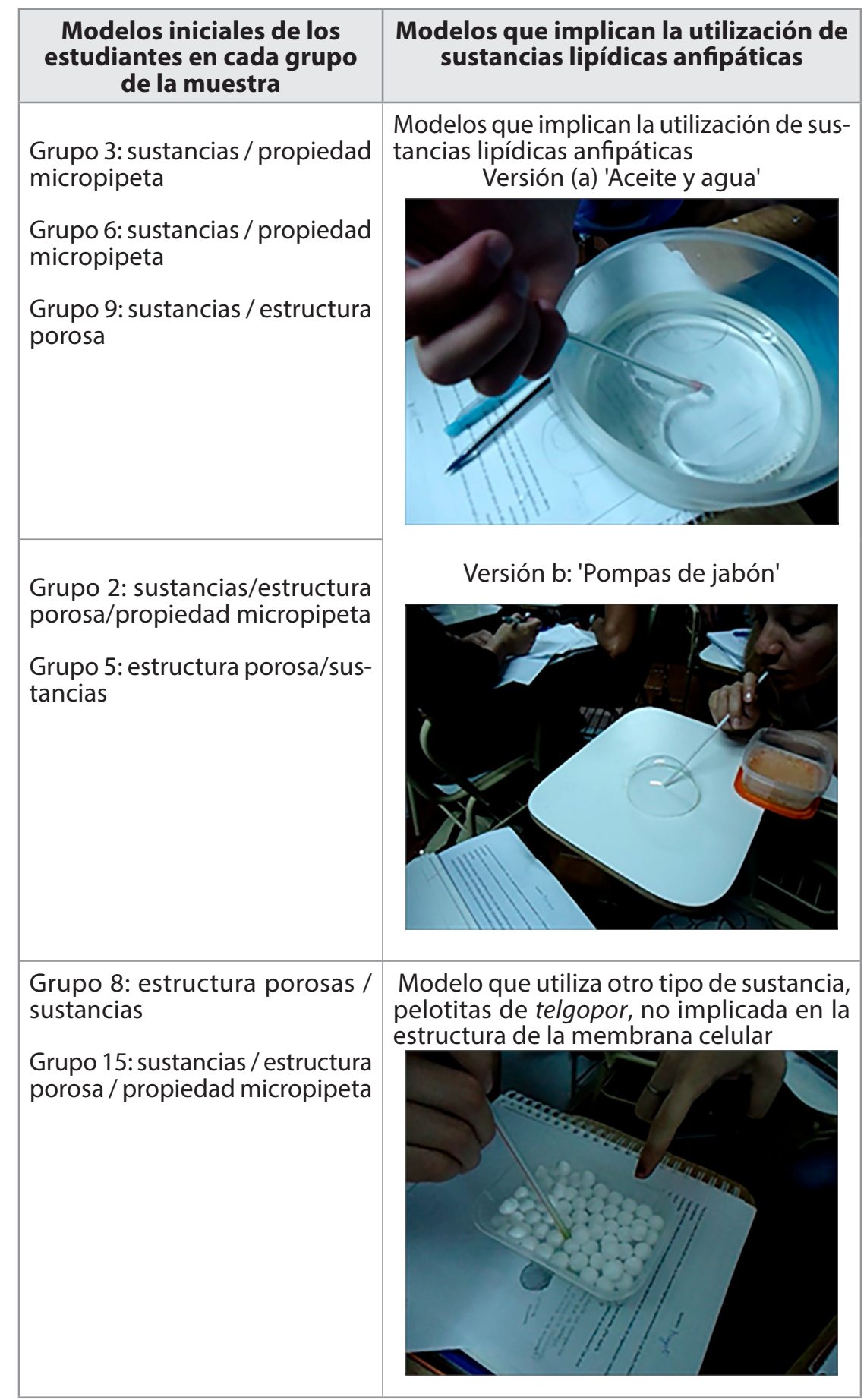

Fuente: Elaborada por los autores a partir de la codificación de las producciones de los estudiantes.

Un aspecto no previsto en la etapa de diseño de la Unidad Didáctica (UD) fue la aparición de las pompas de jabón en dos de los grupos, como análogo material en esta primera etapa de la modelización intermedia. En uno de los grupos de la muestra surgió por la exploración con los diferentes materiales y en el otro fue propuesto por una integrante quien recordaba que era posible intervenir una pompa sin que colapsara. 


\section{b. Modelizaciones utilizando un mediador simbólico}

En la sesión 3, la actividad grupal de lectura del texto que propone la analogía con una pompa de jabón (ver Anexo A) extendió a todos los grupos de la clase la posibilidad de pensar en las pompas de jabón como análogos de la membrana. Mientras las producían y dejaban estables sobre el pupitre para luego intervenirlas, se les pedía que explicaran por qué era posible el ingreso y salida del sorbete sin colapsar. De las ideas expuestas por cada uno de los grupos, fue posible identificar argumentos basados en propiedades macroscópicas del sistema: elástico, flexible, permeable y estable, para justificar el comportamiento de las pompas ante la introducción del sorbete.

Cuadro 4 - Ejemplos de explicaciones sobre el comportamiento de la pompa de jabón

\begin{tabular}{|l|l|}
\hline \multicolumn{1}{|c|}{ Grupos } & \multicolumn{1}{c|}{ Textos explicativos sobre el comportamiento de la pompa de jabón } \\
\hline G 5 & $\begin{array}{l}\text { Creemos que está formada por lípidos lo que hace que esto sea flexible, que mantenga su forma y que } \\
\text { sea estable aunque ingrese la pipeta }\end{array}$ \\
\hline G 8 & $\begin{array}{l}\text { Se puede observar en tres dimensiones, el detergente junto con el agua hace que la estructura sea elástica } \\
\text { y permeable, permitiendo el ingreso de la micropipeta }\end{array}$ \\
\hline G 15 & $\begin{array}{l}\text { La pompa permite que al introducir un sorbete no se rompa. La pared de la pompa es elástica, flexible } \\
\text { y permite que al entrar el sorbete no se rompa }\end{array}$ \\
\hline
\end{tabular}

Fuente: Elaborada por los autores a partir de la codificación de las producciones de los estudiantes.

Las actividades propuestas en el segundo momento de la Sesión 3, de intervenir con un sorbete la pompa, de ligar varias pompas de jabón y gotas de aceite en un recipiente con agua, debían ser ahora traducidas por los alumnos a un nivel molecular utilizando el mediador simbólico propuesto por Quincke: el gráfico de un lípido anfipático (ver Anexo A). Este trabajo presentó importantes dificultades a los estudiantes y fue necesario introducir una actividad en el diseño inicial de la UD para facilitar la transición entre el nivel macro/ observable y la representación gráfica a nivel molecular/abstracta. Se les pidió que, en atención a las dos diferentes propiedades electroquímicas presentes en una molécula de lípido (una zona polar y una zona hidrofóbica), construyeran un modelo que representara cómo se dispondría una pequeña cantidad de ellas si eran colocadas en agua. Luego de intensas discusiones y de ensayos con inconsistencias, todos los grupos lograron aproximar una representación gráfica, afín al modelo de micela.

\section{Modelos de arribo}

Luego de la actividad anterior, se retomó la tarea que había quedado pendiente y la totalidad de los grupos pudo representar a nivel molecular la unión de pompas entre sí, gotitas de aceite y la entrada y salida de la micropipeta a través de la membrana celular. 
Cuadro 5 - Ejemplos de modelos de autoensamblaje utilizando el mediador simbólico

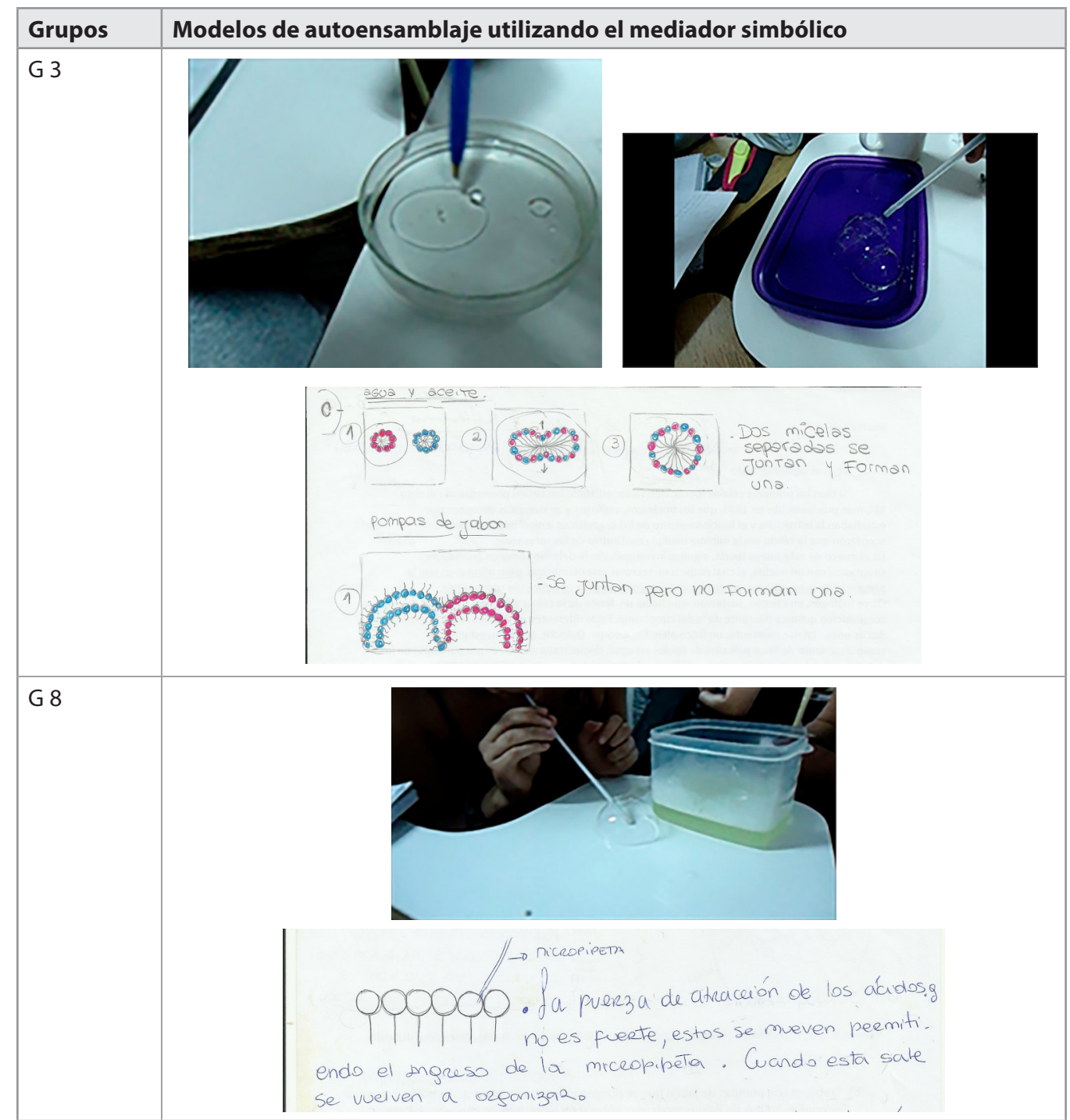

Fuente: Elaborada por los autores a partir de la codificación de las producciones de los estudiantes.

Al comparar las justificaciones que elaboraron los estudiantes luego del trabajo de modelización utilizando el mediador simbólico con las que ofrecieron al inicio de la Sesión 3 para explicar el comportamiento de las pompas, se advierte que prácticamente se dejan de utilizar los conceptos de flexibilidad, elasticidad y el fenómeno es referido en términos de movimiento, reorganización de moléculas, reacomodamiento.

Cuadro 6 - Ejemplos de explicaciones que incorporan el mediador simbólico

\begin{tabular}{|l|l|}
\hline Grupos & \multicolumn{1}{c|}{ Textos explicativos que incorporan el mediador simbólico } \\
\hline G 5 & $\begin{array}{l}\text { Las moléculas que componen las dos gotas se reorganizan para formar una sola, ya que las fuerzas de } \\
\text { atracción entre ellas son débiles }\end{array}$ \\
\hline G 8 & $\begin{array}{l}\text { Hay movimiento. Se pueden mover para sumar otras. La fuerza de atracción de las colitas hidrofóbicas no } \\
\text { es fuerte, esto se mueven permitiendo el ingreso de la micropipeta. Cuando esta sale se vuelven a organizar }\end{array}$ \\
\hline G 15 & $\begin{array}{l}\text { Al mover el agua se unen las gotas de aceite porque sus enlaces son débiles y al abrirse pueden incorporar } \\
\text { más moléculas de aceite formando otro círculo más por las fuerzas de atracción y debe haber movimiento. }\end{array}$ \\
\hline
\end{tabular}

Fuente: Elaborada por los autores a partir de la codificación de las producciones de los estudiantes. 


\section{Discusión y Conclusiones}

Las categorías construidas para dar sentido a los modelos iniciales de los estudiantes fueron también formuladas para los datos obtenidos en la Fase preliminar del ciclo iterativo de implementación de la unidad didáctica. De este modo, pensar en sustancias autosellantes, básicamente aceite, gelatina y agua, o en una estructura porosa para caracterizar el límite de la célula, da lugar a ideas consistentes en el contexto del problema planteado. Al abordar el problema, prácticamente no se hacen referencias al modelo de 'mosaico fluido' teóricamente enseñado en la escuela secundaria. La presencia de más de una hipótesis en algunas de las producciones individuales y la densidad de la descripción gráfica y textual en la mayoría de ellas, tal como se muestra en los registros del cuadro 1, permite inferir que la situación problemática presentada convocó el interés de los estudiantes, que fue significativa y que promovió procesos cognitivos como la construcción de representaciones mentales, de analogías y de argumentaciones a la hora de justificar los modelos iniciales.

La continuación de la modelización al interior de cada grupo implicó procesos de comunicación y negociación de las hipótesis individuales y de selección de las ideas más consistentes y razonables para sostener un análogo concreto que cumpliera con las restricciones que implicaba el fenómeno. En este proceso, las sustancias de naturaleza lipídica, con una sola mención en las ideas iniciales, se implicaron en buena parte de los nuevos modelos. La categoría sustancias lipídicas anfipáticas del cuadro 3, aglutina las producciones basadas en la utilización de sustancias muy cercanas a las propiedades de la membrana celular y sólo se diferencian en el tipo de sistema construido. Las pompas de jabón darían cuenta de las preocupaciones de los alumnos por lograr que, además del autosellado de las sustancias, se obtuvieran estructuras tridimensionales estables para la operación de introducir y sacar objetos a semejanza de una micropipeta y un óvulo.

En el segundo modelo, que los propios alumnos denominaron 'pelotero', la analogía con la membrana celular se estableció en términos de destacar que en el límite de la célula existen unidades que se desplazan y luego se autosellan (fenómeno que se produce por la tensión superficial del agua en la cual se encuentran distribuidas las pelotitas). Si bien este análogo está muy alejado de las propiedades de la célula, se rescata una propiedad central, la auto-organización de las pelotitas, lo que logra satisfacer el requisito. A partir de la introducción de un nuevo punto de vista para analizar el problema, la analogía de Quincke y la presentación de un mediador simbólico: una representación gráfica del lípido anfipático, se dio lugar a un análisis a nivel molecular del comportamiento del modelo 'gota de aceite' y 'pompa de jabón', mediante definiciones gráficas y textuales. Del análisis de los datos surge que adquirieron nuevos significados e implicaron la utilización de un nuevo lenguaje (cuadros 4 y 6) y el desarrollo de argumentaciones más robustas a la hora de explicar la unión de gotas de aceite y de pompas de jabón (cuadro 5). En los dos grupos que propusieron el modelo 'pelotero', la asimilación que hicieron los estudiantes de los lípidos a cada una de las pelotitas que utilizaron, fue inmediata y esto da cuenta de la consistencia que tuvo ese análogo concreto al explicar el fenómeno en la primera etapa de la modelización. En esta línea de análisis vemos que el modelo gráfico producido por G8 en el cuadro 5 es uno de los que explicaron satisfactoriamente la fluidez y el autosellado de la membrana ante la intervención de la micropipeta. Fue propuesto luego del proceso de modelización que utilizó el mediador simbólico de lípido anfipático, pero su definición gráfica es perfectamente compatible con la imagen de sorbete y 'pelotero' que produjeron los grupos G8 y G15. 
En el Anexo B se presenta un resumen cuantificado de la modelización en sus diferentes etapas.

Así, concluimos que:

- En el marco de la construcción de los modelos científicos escolares, la consideración del problema de la manipulación de células con micropipetas para la fertilización in vitro se constituyó como un caso que fue bien resuelto y por lo tanto pasó a constituirse como ejemplar (IZQUIERDO et al., 1999), esto es, como un hecho paradigmático que permitiría conectar significativamente, mediante una representación, el modelo de fluidez y autoensamblaje de la membrana con un fenómeno de la realidad;

- Con la modelización, se avanzó significativamente en la reconsideración teórica del fenómeno, en su construcción como hecho científico escolar y también en el desarrollo de la retórica que implicó interacciones dialógicas significativas y la comunicación de las ideas reformuladas;

- Se pudieron describir los modelos de partida (individuales) y de llegada (grupales) y la etapa de la modelización de las sesiones 1 y 2 constituye una unidad de sentido en sí misma, ya que permitió de manera razonable resolver el problema planteado y podría ser desarrollada en instancias formativas en las cuáles no sea necesario implicar el nivel molecular de organización.

Finalmente, sostenemos que es necesario avanzar en investigaciones en la perspectiva modelos y modelización que, tal como se ha propuesto en este estudio, integren el campo de la formación disciplinar y metacientífica e impliquen el diseño de nuevas unidades didácticas. Estas deberían permitir a los estudiantes ampliar el campo de experiencias para pensar teóricamente diferentes fenómenos del mundo $\mathrm{y}$, simultáneamente, a la propia ciencia. Creemos en el potencial de extender esta propuesta a la formación biológica en general, apuntando a la construcción de una identidad en la formación del profesorado en Biología y, en función del tipo de problemas que se aborden y de su utilidad en la toma de decisiones informadas para intervenir en el mundo, en la posibilidad de incidir más significativamente en cuanto a la mejora de los niveles de alfabetización científica de los ciudadanos (BAHAMONDE, 2014).

\section{Referencias}

ADÚRIZ-BRAVO, A. Una introducción a la naturaleza de la ciencia. Buenos Aires: Fondo de Cultura Económica, 2005.

ADÚRIZ-BRAVO, A. A 'semantic' view of scientific models for science education. Science \& Education, New York, v. 22, n. 7, p. 1593-1611, 2012.

BAHAMONDE, N. Pensar la educación en biología en los nuevos escenarios sociales: la sinergia entre modelización, naturaleza de la ciencia, asuntos sociocientíficos y multirreferencialidad. Bio-grafía, Bogotá, v. 7, n. 3, p. 87-98, 2014. DOI: http://doi.org/dzth

BAHAMONDE, N; GÓMEZ GALINDO, A. Caracterización de modelos de digestión humana a partir de sus representaciones y análisis de su evolución en un grupo de docentes y auxiliares académicos.

Enseñanza de las Ciencias, Barcelona, v. 34 n. 1, p. 129-147. 2016.

BARDIN, L. Análisis de contenido. 3. ed. Madrid: Akal, 1986.

BUCKLEY, B. C. Interactive multimedia and model-based learning in biology. International Journal of Science Education, London, v. 22, n. 9, p. 895-935, 2000. 
CLEMENT, J. Model based learning as a key research area for science education. International Journal of science education, London, v. 22, n. 9, p. 1041-1053, 2000.

CLÉMENT, P. Introducing the cell concept with both animal and plant cells: a historical and didactic approach. Science \& Education, New York, v. 16, p. 423-440, 2007.

DÍAZ DE BUSTAMANTE, J.; JIMÉNEZ ALEIXANDRE, M. El desarrollo de competencias para usar la noción de célula en secundaria. In: CALVO, P.; FONFRÍA, J. (ed.). Recursos didácticos en ciencias naturales. Madrid: Real Sociedad Española de Historia Natural, 2008. p. 169-186.

GIERE, R. La explicación de la ciencia: un acercamiento cognoscitivo. México: Consejo Nacional de Ciencia y Tecnología, 1988.

GILBERT, J.; OSBORNE, R. The use of models in science and science teaching. European Journal of Science Education, London, v. 2 n. 1, p. 3-13, 1980.

GILBERT, J.; JUSTI, R. Models of modelling. In: GILBERT, J.; JUSTI, R. (ed.). Modelling-based teaching in science education. Cham: Springer, 2016. p. 17-40.

GÓMEZ, A. A. La construcción de un modelo de ser vivo en la escuela primaria: una visión escalar. 2005. Tesis (Doctorado en Enseñanza de las Ciencias) - Facultat de Ciències de la Educació, Universitat Autònoma de Barcelona, Barcelona, 2005.

GUTIÉRREZ, R. Lo que los profesores de ciencia conocen y necesitan conocer acerca de los modelos: aproximaciones y alternativas. Bio-grafía, Bogotá, v. 7, n. 13. p. 37-66, 2014.

HIDALGO, C.; DEVÉS, R.; LAGOS, N. Organización molecular de las membranas biológicas. In: LATORRE, R. et al. Biofísica y fisiología celular. Sevilla: Universidad de Sevilla, 1996. p. 3-32.

IDÁRRAGA, A. Enseñanza-aprendizaje del concepto de membrana celular en estudiantes de básica secundaria. 2015. Tesis (Maestría en Enseñanza de de las Ciencias Exactas y Naturales) - Facultad de Ciencias Exactas y Naturales, Universidad Nacional de Colombia, Manizales, 2015.

IZQUIERDO, M.; ESPINET, M.; GARCÍA, M. P.; PUJOL, M. R.; SANMARTÍ, N. Caracterización y fundamentación de la ciencia escolar. Enseñanza de las Ciencias, Barcelona, p. 79-92, 1999. (Número extra).

JUSTI, R. La enseñanza de ciencias basada en la elaboración de modelos. Enseñanza de las Ciencias, Barcelona, v. 24, n. 2, p. 173-184, 2006.

KHAN, S. What's missing in model-based teaching. Journal of Science Teacher Education, London, v. 22, n. 6, p. 535-560, 2011.

LAYING THE FOUNDATION. The fluid mosaic membrane: modeling membrane structure and osmosis. [2004]. Recuperado el 24 mar. 2019 de: https://tinyurl.com/yb3egh8r.

LEDERMAN, N. G. Research on nature of science: reflections on the past, anticipation of the future. Asia-Pacific Forum on Science Learning and Teaching, v. 7, n. 1, p. 2-12, 2006.

LOZANO, E.; BAHAMONDE, N; ADÚRIZ-BRAVO. Análisis histórico-epistemológico sobre los modelos de membrana celular para enseñar biología celular y naturaleza de la ciencia al profesorado. Filosofia e História da Biologia, São Paulo, v.11, n. 1, p. 49-68, 2016.

MARBA, A. Las progresiones del aprendizaje. Alambique: didáctica de las ciencias experimentales, Barcelona, n. 76, p. 71-79, 2014. 
MENGASCINI, A. Propuesta didáctica y dificultades para el aprendizaje de la organización celular. Revista Eureka sobre Enseñanza y Divulgación de la Ciencia, Cádiz, v. 3, n. 3, p. 485-495, 2006.

NERSESSIAN, N. The cognitive basis of model-based reasoning in science. In: CARRUTHERS, P.; STICH, S.; SIEGAL, M. (ed.). The cognitive basis of science. Cambridge: Cambridge University Press, 2002. p. $133-153$.

THE NUFFIELD FOUNDATION. Teaching about science: theoretical models: cell membrane. Leeds: University of Leeds, 2001. Recuperado el 24 mar. 2019 de https://tinyurl.com/y77f9knf

PSILLOS, D.; KARIOTOGLOU, P. Interative design of teaching-learning sequences. London: Springer, 2016.

RAUCHFUSS, H. Chemical evolution and the origin of life. Berlin: Springer, 2008.

REIGELUTH, C.; FRICK, T. Investigación formativa: una metodología para crear y mejorar teorías de diseño. In: REIGELUTH, C. (ed.). Diseño de la instrucción: teorías y modelos: un nuevo paradigma de la teoría de la instrucción. Madrid: Santillana, 1999. v. 2, p. 181-200.

RODRÍGUEZ PALMERO, M. A.; MOREIRA, M. Modelos mentales de la estructura y el funcionamiento de la célula: dos estudios de caso. Investigações em Ensino de Ciências, Porto Alegre, v. 4, n. 2, p. 121 160, 1999.

SANMARTÍ, N. Didáctica de las ciencias en la educación secundaria obligatoria. Madrid: Síntesis Educación, 2002.

TAYLOR, P. Contemporary qualitative research: toward an integral research perspective. In: LEDERMAN, N.; ABELL, S. Handbook of research on science education. New York: Routledge, 2014. p. 38-54.

UNIVERSITY OF UTAH. Genetic Science Learning Center. Build a membrane. Salt Lake City, University of Utah, 2008.

VERHOEFF, R. Towards systems thinking in cell biology education. Utrecht: Universiteit Utrecht, 2003. Recuperado el 24 mar. 2019 de https://tinyurl.com/ya7828fe

ZEIDLER, D.; SADLER, T.; SIMMONS, M.; HOWES, E. Beyond STS: a research based framework for socioscientific issues education. Science Education, Hoboken, v. 89, n. 3, p. 357-377, 2005.

Anexo A - Texto que utilizaron los estudiantes al inicio de la Sesión 3

Si bien las primeras células fueron descriptas en 1665, fue recién promediando el siglo XIX, más precisamente en 1839, que los botánicos, zoólogos y anatomistas europeos que estudiaban la estructura y el funcionamiento de los organismos a nivel microscópico aceptaron que la célula era la mínima unidad constitutiva de los seres vivos. En el marco de esta nueva teoría, algunos investigadores la definían como un grumito de citoplasma con un núcleo, el cual podía o no secretar una membrana, pero afirmaban que la presencia de la membrana no era condición necesaria para su definición como célula. 
Otros biólogos, en cambio, sostenían que había un límite de la célula y además, que éste era de una composición química diferente de la del citoplasma. Estas diferentes posiciones teóricas generaron discusiones. En ese momento, un físico alemán, Georg Quincke, quien investigaba el comportamiento de finas películas de lípidos en agua, demostraba su interés por participar de los debates sobre la naturaleza química de la membrana celular, y en un informe de 1888 escribió:

[...] la superficie plasmática consiste de una membrana fluida muy delgada que envuelve el contenido mucoso y acuoso de la célula en una superficie cerrada, así como una burbuja de jabón encierra al aire. La sustancia de esta membrana es un fluido, que forma gotas en agua y no se mezcla con el agua. Puesto que, de todas las sustancias de origen orgánico conocidas, sólo los aceites muestran esta propiedad, la superficie plasmática debe consistir de aceite graso. (KEPNER, 1979 apud HIDALGO; DEVÉS; LAGOS, 1996, p. 6).

Los jabones con los que se construyen las pompas, incluidas las referenciadas por Quincke, se hace a partir de lípidos saponificables y están formadas por ácidos grasos, los cuales tienen una cabeza polar que es hidrófila, y una cadena carbonada que es hidrófoba. Los químicos acuerdan graficar estos lípidos de la siguiente manera:

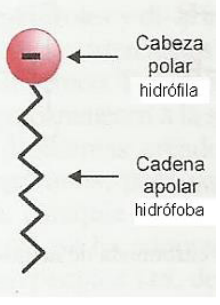

Anexo B - Cuadro resumen de producciones en las diferentes etapas de la modelización

\begin{tabular}{|l|l|l|l|l|l|}
\hline \multicolumn{2}{|c|}{ Modelización inicial. Etapa de trabajo individual } & \multicolumn{2}{|c|}{$\begin{array}{c}\text { Modelización intermedia. } \\
\text { Etapa de trabajo grupal }\end{array}$} & Modelos de arribo \\
\hline $\begin{array}{l}\text { Analogías con sus- } \\
\text { tancias }\end{array}$ & $\begin{array}{l}\text { Referencia a es- } \\
\text { tructuras (poros) }\end{array}$ & $\begin{array}{l}\text { Otras explicaciones } \\
\text { vinculadas a propieda- } \\
\text { des de la micropipeta }\end{array}$ & $\begin{array}{l}\text { Modelos que impli- } \\
\text { can la utilización de } \\
\text { sustancias lipídicas } \\
\text { anfipáticas }\end{array}$ & $\begin{array}{l}\text { Modelo que utiliza otro } \\
\text { tipo de sustancia, no im- } \\
\text { plicada en la estructura } \\
\text { de la membrana celular }\end{array}$ & $\begin{array}{l}\text { Modelizaciones gráficas } \\
\text { y análogos concretos } \\
\text { que explican la fluidez } \\
\text { y el autoensamblaje en } \\
\text { función del problema } \\
\text { planteado }\end{array}$ \\
\hline $\begin{array}{l}51 \% \text { de las men- } \\
\text { ciones }\end{array}$ & $\begin{array}{l}25 \% \text { de las men- } \\
\text { ciones }\end{array}$ & $24 \%$ de las menciones & 5 grupos & 2 grupos & 7 grupos \\
\hline
\end{tabular}

Fuente: elaborado por los autores. 